\title{
Genetic analysis of postoperative recurrence of pancreatic cancer potentially owing to needle tract seeding during EUS-FNB
}

\section{(ㄷ)(1) $\ominus$}

Authors

Hidemasa Kawabata ${ }^{1,2}$, Yuki Miyazawa ${ }^{1}$, Hiroki Sato ${ }^{1}$, Tetsuhiro Okada ${ }^{1,4}$, Akihiro Hayashi ${ }^{1,2}$, Takuya Iwama ${ }^{1}$, Shugo Fujibayashi ${ }^{1}$, Takuma Goto ${ }^{1}$, Junpei Sasajima ${ }^{1}$, Shuhei Takauji ${ }^{1}$, Mikihiro Fujiya1, Yoshihiro Torimoto ${ }^{2}$, Mishie Tanino ${ }^{3}$, Yuko Omori ${ }^{5}$, Yusuke Ono ${ }^{1,4}$, Hidenori Karasaki ${ }^{4}$, Yusuke Mizukami ${ }^{1,4}$, Toshikatsu Okumura ${ }^{1}$

Institutions

1 Department of Medicine

2 Oncology Center

3 Department of Surgical Pathology, Asahikawa Medical University, Asahikawa, Hokkaido, Japan

4 Institute of Biomedical Research, Sapporo-Higashi Tokushukai Hospital, Sapporo, Hokkaido, Japan

5 Department of Investigative Pathology, Tohoku University Graduate School of Medicine, Sendai, Japan

submitted 25.6.2019

ccepted after revision 9.9.2019

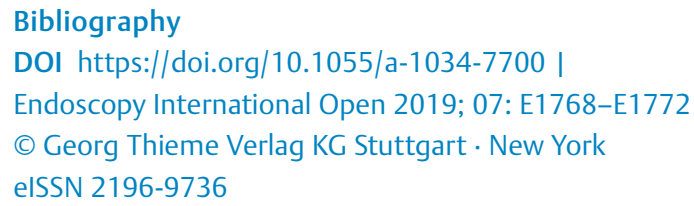

Corresponding author

Yusuke Mizukami, MD, PhD, Division of Gastroenterology and Hematology/Oncology, Department of Medicine, Asahikawa Medical University, 2-1 Midorigaoka Higashi, Asahikawa, Hokkaido 078-8510, Japan

Fax: +81-166682462

mizu@asahikawa-med.ac.jp

\section{Supplementary material}

Online content viewable at:

https://doi.org/10.1055/a-1034-7700
ABSTRACT

Background and study aims Needle tract seeding during endoscopic ultrasound fine-needle biopsy (EUS-FNB) remains a concern. We investigated whether such seeding occurred in a patient with pancreatic ductal adenocarcinoma (PDA).

Patient and methods Surgically resected and EUS-FNBderived specimens were genotyped to determine if a gastric wall tumor that emerged 3 years after curative resection of an early-stage PDA was clonally related to the original tumor.

Results The gastric tumor histologically resembled the primary PDA; the lesions also shared KRAS, SMAD4, and RNF43 mutations. Genotyping of the preoperative EUS-FNB specimen, in which cancer was not detected, nevertheless revealed mutations that were identical to those in the resected primary and recurrent tumors. While the primary PDA had a low frequency of mutant SMAD4, such mutations were highly prevalent in both the EUS-FNB and recurrent tumor specimens.

Conclusions The genetic lineages of sampled tissues from our patient revealed that needle tract seeding may have incidentally occurred when a subset of neoplastic cells within a heterogeneous tumor (i.e., an aggressive clone) was targeted during EUS-FNB.

\section{Introduction}

Pancreatic ductal adenocarcinoma (PDA) has a dismal prognosis. The standard procedure for diagnosing PDA includes tumor tissue sampling using endoscopy; the sensitivity and specificity of endoscopic ultrasound-guided fine needle biopsy (EUSFNB) are over $90 \%$ [1]. Given the utility of EUS in visualizing even small tumors, EUS-FNB has been widely used to obtain samples for the histological analysis of suspected pancreatic le- sions [2,3]. The procedure is particularly useful when the lesion is too small for imaging modalities to ascertain whether a malignancy that warrants surgical intervention is present.

EUS-FNB is a safe examination in most cases, and recurrence owing to needle tract seeding is considered an infrequent event [4]. However, in the event of its occurrence, such procedurerelated dissemination of cancer cells precludes the possibility of a cure even in patients with early-stage PDA [5]. Therefore, physicians need to weigh the risk of performing EUS-FNB in terms of missing an opportunity for curative surgical resection 

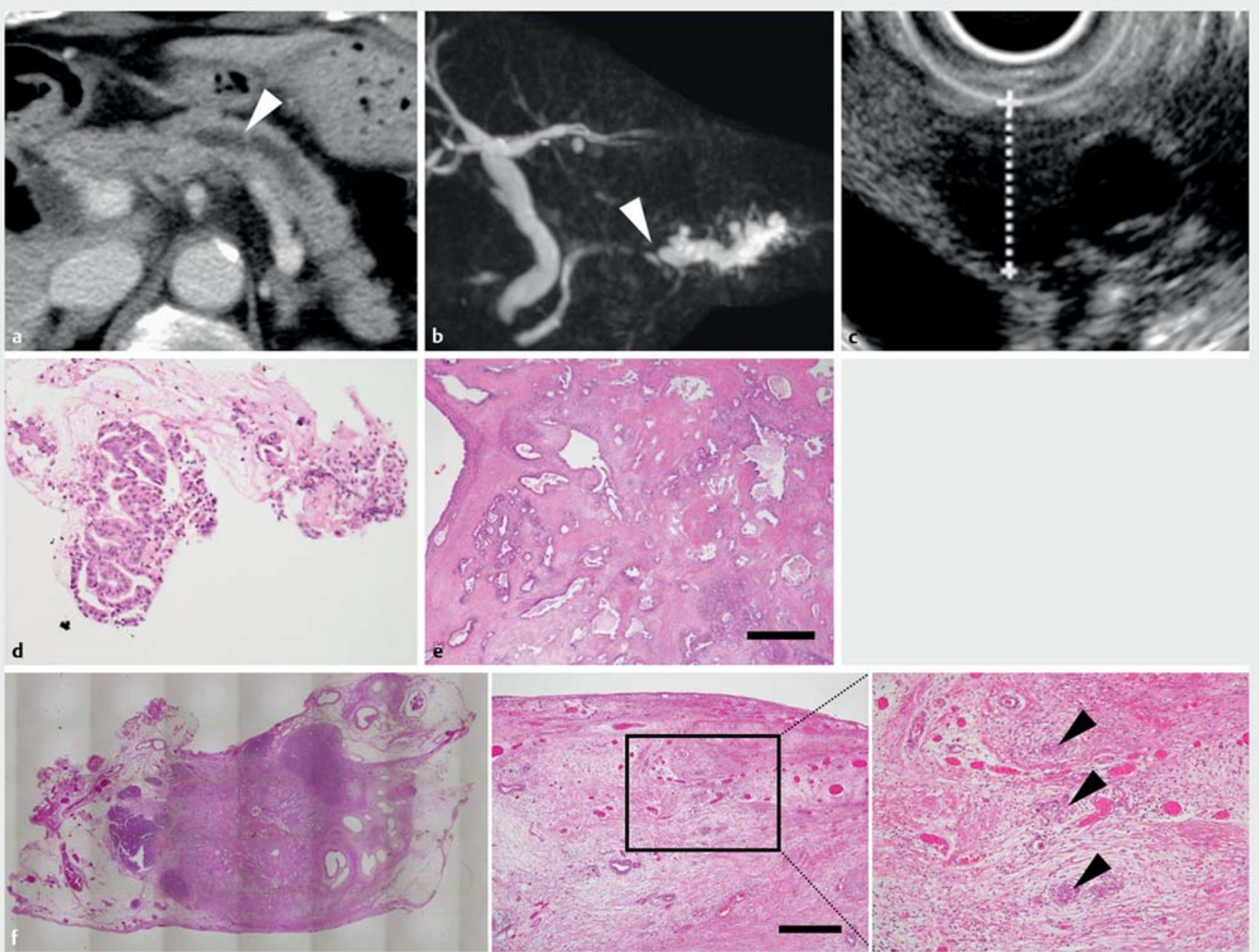

- Fig. 1 Imaging findings of the primary pancreatic tumor and resected specimen. a Computed tomography showed dilation of the main pancreatic duct (MPD; arrowhead) with no sign of a solid tumor. b Magnetic resonance cholangiopancreatography demonstrated an obstruction of the MPD (arrowhead) accompanied by distal dilatation. $\mathbf{c}$ The hypoechoic area was visualized by endoscopic ultrasonography in the body of the pancreas connected to the dilated MPD. Scale bar: $1 \mathrm{~mm}$. $\mathbf{d}$ Endoscopic ultrasound-guided fine-needle biopsy identified few atypical epithelial cells. e Moderately differentiated adenocarcinoma. $\mathbf{f}$ Macroscopic findings for the primary pancreatic ductal carcinoma (left panel). Serosal involvement was not evident (middle panel); however, the lining of the micro-foci of the adenocarcinoma in the granulation tissue was observed (right panel, arrowhead). Scale bar: $100 \mu \mathrm{m}$.

versus causing the iatrogenic acceleration of a potentially aggressive tumor.

Here, we report on a patient with PDA whose resected primary and recurrent tumors, together with endoscopic biopsy specimens from the primary tumor, were subjected to mutational analysis of common PDA-related genes with the goal of determining whether needle tract seeding occurred as a result of EUS-FNB.

\section{Case report}

A 78-year-old woman with no family history of cancer was referred to our hospital because of abdominal pain. Laboratory tests revealed high levels of amylase (139/U/L) and lipase (192 IU/L), but a normal level of carbohydrate antigen 19-9 $(28.0 \mathrm{U} / \mathrm{mL})$. Computed tomography $(\mathrm{CT})$ and magnetic reso- nance imaging showed that the main pancreatic duct (MPD) was dilated to $4.6 \mathrm{~mm}$ with no sign of a solid tumor ( $\mathbf{F i g . 1 a}$, - Fig. 1b). EUS revealed a 10.8-mm hypoechoic area in the body of the pancreas connecting to the dilated MPD ( $>$ Fig. 1c).

For pathological assessment, EUS-FNB using a 22-gauge needle (EchoTip ProCore HD Ultrasound Biopsy Needle, Cook Japan Inc., Tokyo, Japan) was performed. Few atypical epithelial cells were identified; however, no malignancy was verified histologically ( $\triangleright$ Fig. $1 \mathbf{d}$ ), and abnormal p53 staining was not observed. Serial sampling of the pancreatic duct via a transpapillary route was then performed, which also revealed no malignancy.

Subsequently, however, dilatation of the MPD continued to progress concomitant with rising serum amylase and lipase levels, and it was recommended that the patient undergo surgical resection of the pancreas after an invasive PDC was suspected. 

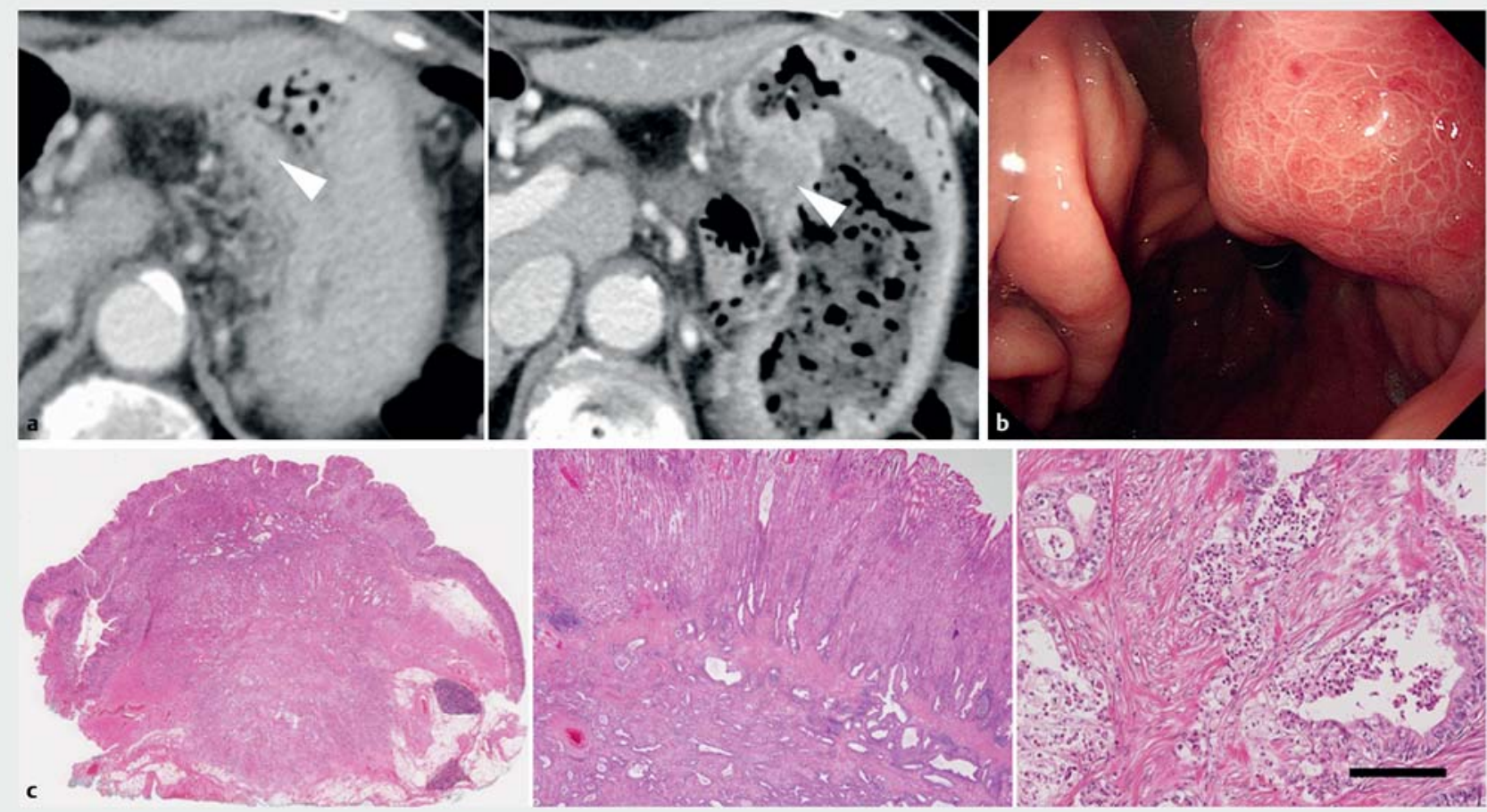

- Fig. 2 Recurrence of pancreatic ductal carcinoma in the gastric wall. a, b A submucosal tumor $(25 \mathrm{~mm})$ in the posterior wall of the stomach was demonstrated by computed tomography (CT) (a, right) and esophagogastroduodenoscopy (b). CT performed 1 year before recurrence is also shown (left). Arrowheads indicate the abnormal mass in the gastric wall. c The tumor was histologically identical to the primary pancreatic ductal carcinoma. Scale bars: $100 \mu \mathrm{m}$.

The patient underwent distal pancreatectomy for curative resection of the PDA, achieving a negative surgical margin ( $\vee$ Fig. 1e); the tumor was determined to be a moderately differentiated adenocarcinoma. There was no sign of serosal involvement; however, adenocarcinoma micro-foci in the granulation tissue that were non-contiguous with the primary tumor were observed ( $\triangleright$ Fig. 1f). Lymphovascular invasion was not histologically evident. In addition to the stage I PDA, another welldifferentiated neuroendocrine tumor $(4 \mathrm{~mm})$ was found incidentally (Supplementary Fig. 1a).

Three years after resection of the primary PDA, a 25-mm submucosal tumor in the posterior wall of the stomach was detected ( $\triangleright$ Fig. 2a; right panel, and $\triangleright$ Fig. 2 b). A small mass was also detected retrospectively 2 years after resection upon reexamination of the contrast-enhanced computed tomography image ( $\triangleright$ Fig. 2, left panel). EUS-FNB-obtained tissue specimens from the gastric tumor and from the recurred PDA were highly suspected to be histologically identical. Partial resection of the stomach was then performed, following which the tumor was histologically confirmed to be a recurrence of the PDA ( $\vee$ Fig.2c). Additional small tumors in the gastric wall were also found during the surgery, and were confirmed to be gastrointestinal stromal tumors following their resection (Supplementary Fig. 1b).

To determine whether needle tract seeding due to EUS-FNB occurred, we performed genetic analyses of the tissue specimens that were surgically resected or obtained via biopsy/cy- tology to identify any somatic mutations frequently found in PDA (see Supplementary Methods). Targeted amplicon sequencing revealed KRAS G12D and SMAD4 W323X mutations in the primary tumor, recurrent tumors, and EUS-FNB specimens ( $>$ Table 1). On immunohistochemistry of these samples, SMAD4 exhibited a mosaic staining pattern with retained, reduced, and absent expression observed in the primary tumor ( $\vee$ Fig.3). On the other hand, the majority of the tumor cells exhibited a loss of SMAD4 expression in both the atypical cells obtained by EUS and the recurrent gastric wall tumor.

Biopsy/cytology samples collected via the transpapillary route from the primary PDA, all of which were pathologically negative for cancer cells, were also utilized if found to be useful for analyzing tumor-associated mutations. Given the very low DNA yield from these samples, sequencing could not be performed using endoscopic pancreatic biopsy and cytology specimens, and digital PCR assays targeting KRAS G12D and G12V as well as SMAD4 W323X variants were performed instead ( $\triangleright$ Table 1). Although KRAS mutations were present in some of the samples, only wild-type SMAD4 was detected. Written informed consent was obtained from the patient before performing the genetic study and for publication of this case report. 
Table 1 Results of sequencing of the surgically resected and EUS-FNB specimens.

\begin{tabular}{|c|c|c|c|c|c|c|c|c|c|}
\hline Specimens & $\begin{array}{l}\text { Mean } \\
\text { depth }\end{array}$ & $\begin{array}{l}\text { Unifor- } \\
\text { mity }\end{array}$ & $\begin{array}{l}\text { Target } \\
\text { base } \\
\text { coverage } \\
\text { at } 100 \times \\
\text { (\%) }\end{array}$ & $\begin{array}{l}\text { Target } \\
\text { base } \\
\text { coverage } \\
\text { at } 500 \times \\
(\%)\end{array}$ & KRAS & TP53 & SMAD4 & CDKN2A & Other mutations (\%) \\
\hline EUS-FNB & 3,014 & 0.889 & 98.7 & 89.9 & G12D (49.8) & WT & W323X (49.1) & WT & RNF43 I186F ${ }^{1}(21.2)$ \\
\hline $\mathrm{EPB}^{2}$ & - & - & - & - & G12D (2.1) & - & WT & - & - \\
\hline Primary PDA & 2,755 & 0.952 & 98.5 & 96.3 & G12D (13.8) & WT & W323X (3.5) & WT & RNF43 I186F ${ }^{1}(16.2)$ \\
\hline PNET & 3,383 & 0.946 & 99.2 & 96.0 & WT & WT & WT & WT & ND \\
\hline $\begin{array}{l}\text { Recurrent } \\
\text { tumor }\end{array}$ & 3,307 & 0.902 & 98.5 & 94.7 & G12D (18.8) & WT & W323X (22.7) & WT & RNF43 I186F ${ }^{1}(22.4)$ \\
\hline GIST & 2,763 & 0.930 & 98.7 & 94.7 & WT & WT & WT & WT & ND \\
\hline \multicolumn{10}{|c|}{$\begin{array}{l}\text { EUS-FNB, endoscopic ultrasound-guided fine-needle biopsy; EPB, endoscopic pancreatic biopsy; GIST, gastrointestinal stromal tumor; ND, not detected; PDA, pan- } \\
\text { creatic ductal adenocarcinoma; PNET, pancreatic neuroendocrine tumor. } \\
{ }^{1} \text { The COSMIC (http://cancer.sanger.ac.uk/cosmic) and ClinVar (https://www.ncbi.nlm.nih.gov/clinvar/) databases were used to classify variants of unknown signifi- } \\
\text { cance. }\end{array}$} \\
\hline
\end{tabular}

\section{Discussion}

Our study yielded genetic evidence that obtaining tumor cell samples via EUS-FNB for purposes of definitive diagnosis of pancreatic cancer resulted in needle tract seeding. It is unclear if specific tumor types have a greater propensity for such seeding, and effective protocols aimed at preventing dissemination of cells have not been established. Multiple punctures during EUS-FNB are performed to obtain sufficient specimens for histological diagnosis; however, the chances of tumor cell dissemination may be increased [6]. To maximize the benefit of EUS-FNB while minimizing the risk thereof, this diagnostic approach could be omitted in patients with small tumors that exhibit no extra-pancreatic invasion, as such individuals are candidates for potentially curative treatments.

Presence of KRAS mutation alone may not be sufficient to trace tumor cell clonality during PDA development and recurrence because hotspots are enriched in codons 12 and 13 . When KRAS G12D and G12V are found in multiple lesions, it is not easy to determine if they are clonally related or developed independently $[7,8]$. We therefore performed targeted sequencing to search for genes that are commonly mutated in human PDA [9], wherein we revealed a pathogenic mutation in SMAD4 across the specimens. However, genetic tracing does not confirm that EUS-FNB was indeed responsible for tumor dissemination because metastasis to the gastric wall could also have occurred via direct invasion.

In the primary PDA, the mutated allele frequency of SMAD4 was much smaller than that of KRAS, which indicated intratumor heterogeneity. Of note, the mutation profile obtained via EUS-FNB revealed equal levels of mutated alleles in KRAS and $S M A D 4$, suggesting that the sampled tissue represented a tumor region harboring both mutations, whereas the pancreatic duct biopsy obtained during extended radical resection contained a mutation in KRAS but not SMAD4. Endoscopic biopsies may be less informative when assessing the mutational burdens and heterogeneities because they provide only locoregional information (unless multiple extractions from different areas of the tumor are performed) [9]. In our patient, the mutation-enriched tumor cells collected by EUS-FNB consistently exhibited high mutational burdens of both KRAS and SMAD4, which were observed in the recurrent gastric wall tumor. Subclones of the primary tumor that are more genetically volatile may facilitate needle track dissemination, although the possibility of spontaneous metastasis cannot be excluded. However, we considered the latter scenario unlikely since micro-foci of the adenocarcinoma in the granulation tissue were observed; moreover, neither serosal nor lymphovascular invasion were histologically evident in the resected primary tumor specimen. Neither the pancreatic neuroendocrine tumor nor the gastrointestinal stromal tumor that were incidentally found during surgical resection shared the mutations found in the primary and recurrent PDAs, suggesting that the non-epithelial tumors were clonally unrelated to these PDAs.

If an invasive procedure for curatively resectable (earlystage) PDA is to be avoided, a liquid biopsy can be an alternative noninvasive test for KRAS mutations [10]. We previously demonstrated successful genetic testing of fresh pancreatic juice in a patient with early-stage PDA for whom histological assessment had failed to detect a malignancy [11]. Such assays have variable sensitivities and specificities depending on the source of the sample (i.e., plasma, duodenal/pancreatic juice, or urine), and reliable protocols for sampling, storing, preparing, and assaying nucleic acids need to be established [12]. When combined with modern imaging technologies, such genetic tests are promising tools that allow the forgoing of invasive procedures, thereby avoiding the risk of false negative results when using current endoscopic techniques. 


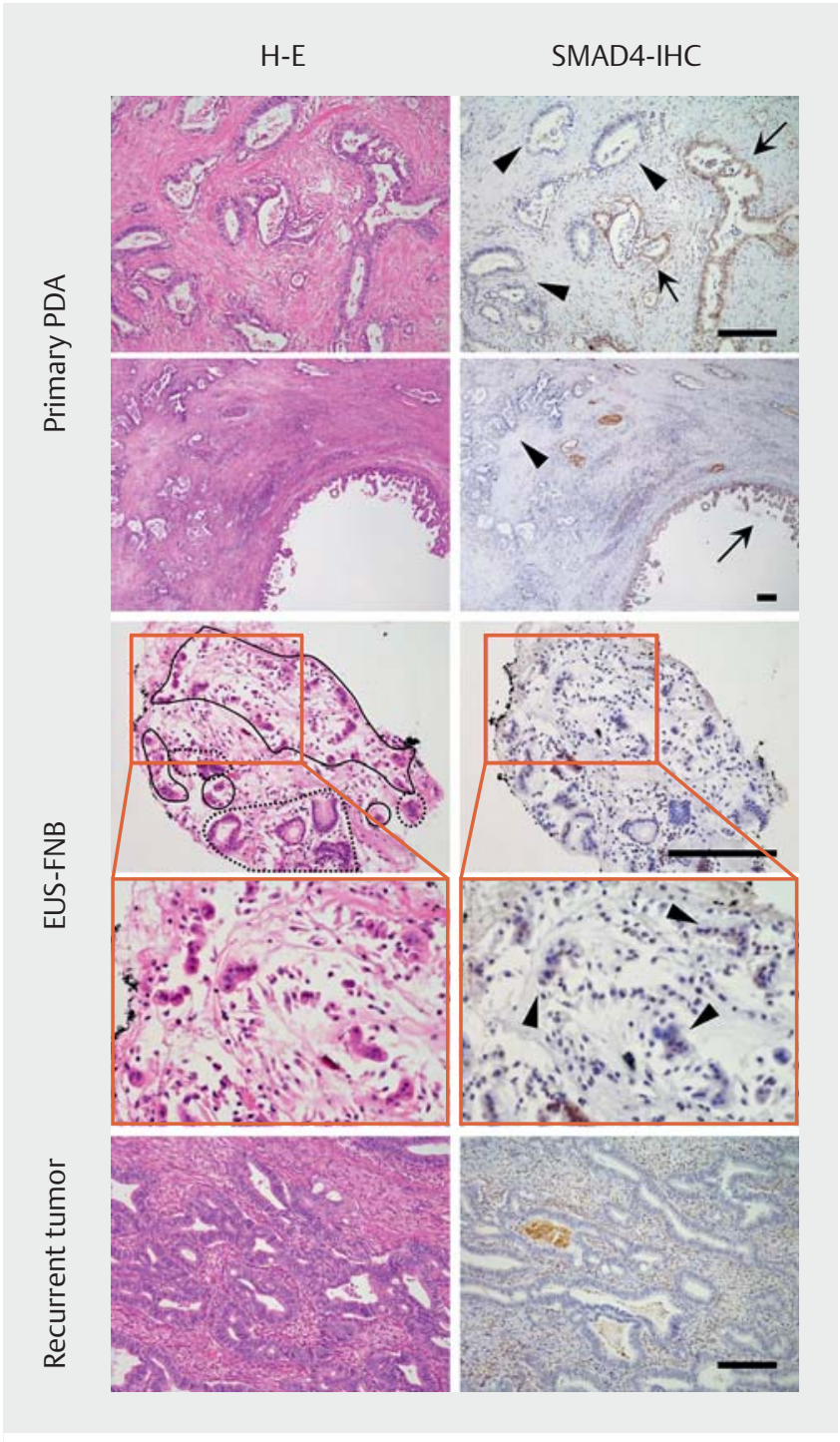

- Fig. 3 Immunostaining for SMAD4 in the surgically resected and endoscopic ultrasound-fine needle biopsy (EUS-FNB) specimens. SMAD4 staining was reduced (arrows) or absent (arrowheads) in the surgically resected specimen (upper panel). Loss of SMAD4 expression was noted in the majority of the tumor cells in the EUSFNB specimens (middle) as well as in the recurrent gastric wall tumor (bottom). Areas marked with a solid line in the middle panel indicate atypical cells suggestive of adenocarcinoma, while dashed lines denote gastric foveolar epithelia. Scale bars: $200 \mu \mathrm{m}$.

\section{Conclusion}

In conclusion, our genetic assessment revealed evidence of a tissue sampling-related recurrence of pancreatic cancer, where needle tract seeding appeared to have occurred in a patient with a heterogeneous tumor that was sampled using EUS-FNB. Less invasive collection of a minimal amount of tissue combined with genetic testing may be a safer alternative to histopathological sampling of early-stage PDA.

\section{Acknowledgements}

The authors thank Munehiko Ogata and Yuko Hayakawa (Sapporo-Higashi Tokushukai Hospital) for tissue sample preparation and genetic analysis.

\section{Competing interests}

Research funding was provided by Hitachi High-Technologies Corporation.

References

[1] Chen G, Liu S, Zhao Y et al. Diagnostic accuracy of endoscopic ultrasound-guided fine-needle aspiration for pancreatic cancer: a meta-analysis. Pancreatology 2013; 13: 298-304

[2] Uehara H, Ikezawa K, Kawada $\mathrm{N}$ et al. Diagnostic accuracy of endoscopic ultrasound-guided fine needle aspiration for suspected pancreatic malignancy in relation to the size of lesions. J Gastroenterol Hepatol 2011; 26: 1256-1261

[3] Crinò SF, Conti Bellocchi MC, Bernardoni L et al. Diagnostic yield of EUS-FNA of small $(\leq 15 \mathrm{~mm})$ solid pancreatic lesions using a 25 -gauge needle. Hepatobiliary Pancreat Dis Int 2018; 17: 70-74

[4] Wang KX, Ben QW, jin ZD et al. Assessment of morbidity and mortality associated with EUS-guided FNA: a systematic review. Gastrointest Endosc 2011; 73: 283-290

[5] Moutinho-Ribeiro P, Iglesias-Garcia J, Gaspar R et al. Early pancreatic cancer - The role of endoscopic ultrasound with or without tissue acquisition in diagnosis and staging. Dig Liver Dis 2019; 51: 4-9

[6] Minaga K, Takenaka M, Katanuma A et al. Needle tract seeding: An overlooked rare complication of endoscopic ultrasound-guided fineneedle aspiration. Oncology 2017; 93: (Suppl. 01): 107-112

[7] Patra KC, Bardeesy N, Mizukami Y. Diversity of precursor lesions for pancreatic cancer: The genetics and biology of intraductal papillary mucinous neoplasm. Clin Transl Gastroenterol 2017; 8: e86

[8] Felsenstein M, Noe M, Masica DL et al. IPMNs with co-occurring invasive cancers: neighbours but not always relatives. Gut 2018; 67: 1652-1662

[9] Omori Y, Ono Y, Tanino M et al. Pathways of progression from intraductal papillary mucinous neoplasm to pancreatic ductal adenocarcinoma based on molecular features. Gastroenterology 2019; 156: $647-661$ e2

[10] Bernard V, Kim DU, San Lucas FA et al. Circulating nucleic acids are associated with outcomes of patients with pancreatic cancer. Gastroenterology 2019; 156: 108-118 e4

[11] Okada T, Iwano H, Ono Y et al. Utility of "liquid biopsy" using pancreatic juice for early detection of pancreatic cancer. Endosc Int Open 2018; 6: E1454-E1461

[12] Suenaga M, Yu J, Shindo K et al. Pancreatic juice mutation concentrations can help predict the grade of dysplasia in patients undergoing pancreatic surveillance. Clin Cancer Res 2018; 24: 2963-2974 